\title{
Solvability of linear differential algebraic equations with properly stated leading terms
}

\author{
Roswitha März
}

\begin{abstract}
In this paper, general solvability statements on linear continuous coefficient differential algebraic equations with properly stated leading terms are derived by means of decoupling projector functions decomposing the differential algebraic equation into its characteristic components.
\end{abstract}

Keywords: Differential algebraic equations, regularity, tractability index, initial value problems

AMS subject classification: $34 \mathrm{~A} 09,34 \mathrm{~A} 30,34 \mathrm{~A} 12$

\section{Introduction}

This paper is a continuation of the approach by [1] to characterize a class of linear differential algebraic equations (DAEs) of the form

$$
A(D x)^{\prime}+B x=q
$$

with continuous coefficients $A, D, B$ analytically exactly.

While an index characterization and the proof of invariance under transformation and refactorization were in the center of interest in [1], the present paper aims at revealing the exact inner mathematical structure and the solvability of DAEs with tractability index $\mu$ by means of partitioning and decoupling projectors.

In distinction to the various DAE concepts available (cf. [2] for a comprehensive overview) great store is set by low resp. exact smoothness conditions here. We do not assume the coefficients to have first and higher derivatives. However, as described in detail in [1], in this context constant dimensions play an important role for a series of subspaces.

The paper is divided into three further sections. Section 2 provides the necessary tools (matrix function sequence, index definition) from [1]. They are completed by the new and important realization on the invariance of special subspaces (Theorem 2.3). In Section 3, we reveal by means of projectors that and in what way each regular DAE can be partitioned into an inherent regular ODE for the dynamical component and a system of explicit equations defining the other components, in which necessary differentiations of already available components have to be carried out, as expected. Finally, initial value problems (IVPs) can be formulated in such a way that statements on the uniqueness and solvability will be possible.

Section 4 is devoted to the problem in how far the system of non-dynamical components can be completely decoupled from the inherent regular ODE. Should this be possible, we will obtain clear and simple solvability statements (Theorem 4.4) as well as an exact description of the function spaces for the admissible right-hand sides $q$ in (1.1). This allows for a description of the canonical subspaces, for instance of the exact geometrical locus of solution for the homogeneous DAE. On 
this background, it will become apparent that, for standard solvability assertions, some necessary smoothness conditions may also concern the DAE coefficients.

Furthermore, the exact knowledge of the function spaces of the admissible right-hand sides provides causality conditions for control problems (Theorem 4.8).

Technically expensive proofs have been placed in the appendices A and B.

\section{Fundamentals}

We consider equations

$$
A(t)(D(t) x(t))^{\prime}+B(t) x(t)=q(t), \quad t \in \mathcal{I}
$$

with continuous matrix coefficients $A(t) \in L\left(\mathbb{R}^{n}, \mathbb{R}^{m}\right), D(t) \in L\left(\mathbb{R}^{m}, \mathbb{R}^{n}\right), B(t) \in L\left(\mathbb{R}^{m}\right)$, $t \in \mathcal{I}, \mathcal{I} \subseteq \mathbb{R}$ an interval, and with properly stated leading term. The right-hand side $q(t) \in \mathbb{R}^{m}$ depends, at least continuously, on $t$. We are looking for continuous functions $x: \mathcal{I} \rightarrow \mathbb{R}^{m}$ that have a continuously differentiable product $D x: \mathcal{I} \rightarrow \mathbb{R}^{n}$, and which satisfy equation (2.1) for all $t \in \mathcal{I}$.

The leading term is said to be stated properly if the matrix functions $A$ and $D$ are well matched in the sense that the decomposition

$$
\operatorname{ker} A(t) \oplus i m D(t)=\mathbb{R}^{n}, \quad t \in \mathcal{I},
$$

is valid and both subspaces are spanned by basis functions that are continuously differentiable on $\mathcal{I}$ (cf. [3], [4]). In the consequence, there is a uniquely determined projector function $R \in C^{1}\left(\mathcal{I}, L\left(\mathbb{R}^{n}\right)\right)$ that realizes the decomposition $(2.2)$, that is, we have $R(t)^{2}=R(t), i m R(t)=$ $\operatorname{im} D(t), \operatorname{ker} R(t)=\operatorname{ker} A(t)$, for $t \in \mathcal{I}$. Observe that $A(t)$ and $D(t)$ have common constant rank on $\mathcal{I}$. Additionally, we use functions $D^{-} \in C\left(\mathcal{I}, L\left(\mathbb{R}^{n}, \mathbb{R}^{m}\right)\right)$ satisfying the conditions

$$
D(t) D(t)^{-} D(t)=D(t), D(t)^{-} D(t) D(t)^{-}=D(t)^{-}, D(t) D(t)^{-}=R(t), \quad t \in \mathcal{I},
$$

i.e., pointwise generalized inverses of $D$. We stress that (2.3) does not define $D^{-}$uniquely (e.g. [5]). However, if we fix an additional projector function $Q_{0} \in C\left(\mathcal{I}, L\left(\mathbb{R}^{m}\right)\right)$ that projects pointwise onto $\operatorname{ker} D(t)$, then the fourth condition

$$
D(t)^{-} D(t)=I-Q_{0}(t), t \in \mathcal{I},
$$

added to (2.3) makes the generalized inverse uniquely determined. We shall take advantage of this fact. Since the decomposition (2.2) implies that $\operatorname{ker} A(t) D(t)=\operatorname{ker} D(t)$, we may operate with $Q_{0}(t)$ being nullspace projectors for the product $A(t) D(t)$.

Next we construct a special sequence of matrix functions and subspaces to be used later on for index characterization and system decoupling. The argument $t$ is mostly dropped. Then the given relations are meant pointwise on $\mathcal{I}$. Starting from the coefficients $A, D, B$ of equation (2.1) we form

$$
\begin{aligned}
& G_{0}=A D, \quad N_{0}=\operatorname{ker} G_{0}, \quad B_{0}=B, \\
& Q_{0}^{2}=Q_{0}, \quad i m Q_{0}=N_{0}, \quad P_{0}=I-Q_{0},
\end{aligned}
$$

and, for $i \geq 0$,

$$
\begin{aligned}
G_{i+1} & =G_{i}+B_{i} Q_{i}, N_{i+1}=\operatorname{ker}_{i+1} \\
Q_{i+1}^{2} & =Q_{i+1}, \quad i m Q_{i+1}=N_{i+1}, P_{i+1}=I-Q_{i+1} \\
B_{i+1} & =B_{i} P_{i}-G_{i+1} D^{-}\left(D P_{0} \cdots P_{i+1} D^{-}\right)^{\prime} D P_{0} \cdots P_{i} .
\end{aligned}
$$


The expressions (2.6) and (2.8) mean that corresponding projectors $Q_{i}(t) \in L\left(\mathbb{R}^{m}\right)$ onto $N_{i}(t)$, $t \in \mathcal{I}, i \geq 0$, are introduced. When using (2.9) we have to take care of the existence of the involved derivative.

Definition 2.1 ([1]): Equation (2.1) is called a regular DAE or a regular DAE with tractability index $\mu$ if there is a sequence (2.5) - (2.9) such that, for $i \geq 0$,

(a) $G_{i}(t)$ has constant rank $r_{i}$ on $\mathcal{I}$,

(b) $N_{0} \oplus \cdots \oplus N_{i} \subseteq \operatorname{ker} Q_{i+1}$,

(c) $Q_{i} \in C\left(\mathcal{I}, L\left(\mathbb{R}^{m}\right)\right), D P_{0} \cdots P_{i} D^{-} \in C^{1}\left(\mathcal{I}, L\left(\mathbb{R}^{n}\right)\right)$,

and $r_{\mu-1}<r_{\mu}=m$.

Clearly, the flexible part of the sequence (2.5) - (2.9) are the projectors. Instead of saying that there is a sequence (2.5) - (2.9) suitable in the sense of Definition 2.1, we can agree to say that there are appropriate projectors $Q_{0}, Q_{1}, \ldots, Q_{\mu-1}$ for that. Note that $r_{\mu}=m$ implies $Q_{\mu+i}=0, G_{\mu+i}=G_{\mu}$, for $i \geq 0$, i.e., the sequence becomes stationary.

Condition (b) in Definition 2.1 is discussed in [1] as a necessary one for regularity. The benefit of this relation are special properties of the projectors and projector products. In particular, it holds that

$$
\begin{gathered}
Q_{i} Q_{j}=0, \quad j=0, \ldots, i-1, \quad Q_{i}=Q_{i} P_{0} \cdots P_{i-1}, \\
\left(P_{0} \cdots P_{i}\right)^{2}=P_{0} \cdots P_{i}, \operatorname{ker}\left(P_{0} \cdots P_{i}\right)=N_{0} \oplus \cdots \oplus N_{i}, \\
\left(D P_{0} \cdots P_{i} D^{-}\right)^{2}=D P_{0} \cdots P_{i} D^{-}, \\
k e r Q_{i}=\operatorname{ker} P_{0} \cdots P_{i-1} Q_{i} \\
\operatorname{ker}\left(D P_{0} \cdots P_{i} D^{-}\right)=D P_{0} \cdots P_{i-1} N_{i} \oplus \cdots \oplus D P_{0} N_{1} \oplus \operatorname{ker} A .
\end{gathered}
$$

The matrix functions $G_{i}$ resulting from (a),(b),(c) are continuous.

The differentiability condition in (c) ensures that all terms in the decomposition of the $C^{1}$ function $R$ belong to $C^{1}$, too, these are

$$
R=D D^{-}=D P_{0} D^{-}=D P_{0} \cdots P_{i} D^{-}+D P_{0} \cdots P_{i-1} Q_{i} D^{-}+\cdots+D P_{0} Q_{1} D^{-} .
$$

In other words, the subspace $i m D$ that has a continuously differentiable base is consecutively decomposed into further such subspaces. As we shall realize below, this corresponds to characteristic parts of the solution.

Although the matrix functions $G_{i}$ clearly depend on the special choice of the projectors within the scope of Definition 2.1, regularity with index $\mu$ does not so. This is proved in [1], where regularity with index $\mu$ is also shown to remain invariant under regular transformations and refactorizations.

Definition 2.2 The sequence (2.5)-(2.9) is said to be admissible up to $k \in \mathbb{I N}$ (or the projector functions $Q_{0}, \ldots, Q_{k}$ are admissible) if, for $i=0,1, \ldots, k$,

(a) $\operatorname{rank}_{i}(t)=r_{i}, t \in \mathcal{I}$,

(b) if $i \geq 1$, then $N_{0} \oplus \cdots \oplus N_{i-1} \subseteq \operatorname{ker} Q_{i}$,

(c) $Q_{i} \in C\left(\mathcal{I}, L\left(\mathbb{R}^{m}\right)\right), \quad D P_{0} \cdots P_{i} D^{-} \in C^{1}\left(\mathcal{I}, L\left(\mathbb{R}^{n}\right)\right)$. 
Theorem 2.3 The subspaces $i m G_{i}$ and $N_{0} \oplus \cdots \oplus N_{i}, i=0,1, \ldots k$, do not at all depend on the special choice of admissible projector functions $Q_{0}, \ldots Q_{k}$.

\section{Proof:}

Take two admissible sequences $G_{j}$ with $Q_{j}, j=0, \ldots k$, and $\bar{G}_{j}$ with $\bar{Q}_{j}, j=0, \ldots k$, and look for relations. We have $G_{0}=\bar{G}_{0}, N_{0}=\bar{N}_{0}, B_{0}=\bar{B}_{0}$, further $\bar{D}^{-}=\bar{D}^{-} D \bar{D}^{-}=\bar{D}^{-} R=\bar{D}^{-} D D^{-}=$ $\bar{P}_{0} D^{-}$. Derive $\bar{G}_{1}=\bar{G}_{0}+\bar{B}_{0} \bar{Q}_{0}=G_{0}+B_{0} \bar{Q}_{0}=G_{0}+B_{0} Q_{0} \bar{Q}_{0}=G_{1}\left(I+Q_{0} \bar{Q}_{0} P_{0}\right)$. Since $I+Q_{0} \bar{Q}_{0} P_{0}$ is a nonsingular factor, it follows that $i m G_{1}=i m \bar{G}_{1}, N_{1}=\left(I+Q_{0} \bar{Q}_{0} P_{0}\right) \bar{N}_{1}, N_{0}+N_{1}=\bar{N}_{0}+\bar{N}_{1}$, hence, $N_{0} \oplus N_{1}=\bar{N}_{0} \oplus \bar{N}_{1}$.

In case of $k=1$ we are done. By careful technical calculations given in Appendix A, we verify factorizations $\bar{G}_{i}=G_{i} Z_{i}$ with nonsingular factors

$$
Z_{i}=\left(I+Q_{i-1} \bar{Q}_{i-1} P_{i-1}+\sum_{j=0}^{i-2} Q_{j} Z_{i j} P_{0} \cdots P_{i-2}\right) Z_{i-1} .
$$

Again, it follows that $i m \bar{G}_{i}=i m G_{i}, N_{i}=Z_{i} \bar{N}_{i}, N_{0} \oplus \cdots \oplus N_{i}=\bar{N}_{0} \oplus \cdots \oplus \bar{N}_{i}, i=2, \ldots, k . \diamond$

Corollary 2.4 For a regular DAE (2.1) with tractability index $\mu$, the subspace

$$
N_{\text {can } \mu}=N_{0} \oplus \cdots \oplus N_{\mu-1}
$$

has dimension $\mu m-r_{0}-\ldots-r_{\mu-1}$, and it is invariant of the choice of the admissible projectors $Q_{0}, \ldots, Q_{\mu-1}$.

The subscript of $N_{\text {can } \mu}$ indicates that this is a canonical subspace for the index $\mu$ DAE.

Corollary 2.5 For constant matrices $A, D, B$ that form a regular matrix pencil $\lambda G_{0}+B_{0}$ with Kronecker index $\mu$, the subspace $N_{\text {can } \mu}$ coincides with the infinite eigenspace of the pencil.

\section{Proof:}

In [6], special so-called canonical projectors $Q_{j}, j=0, \ldots \mu-1$, are chosen for constant matrices to obtain, with $P_{0} \cdots P_{\mu-1}$, the spectral projection onto the finite eigenspace along the infinite one. These canonical projectors are admissible ones too, hence the assertion follows from Theorem 2.3. $\diamond$

\section{Decoupling a regular DAE into its characteristic parts}

Now we deal with regular DAEs (2.1) that have tractability index $\mu$. We try to realize the inherent structure by means of projections. We do not transform the unknown function, but we decompose it into characteristic parts. First of all, we multiply (2.1) by $G_{\mu}^{-1}$ (cf. [1]). The resulting equivalent version of $(2.1)$ is

$$
\begin{aligned}
& P_{\mu-1} \cdots P_{0} D^{-}(D x)^{\prime}+G_{\mu}^{-1} B P_{0} \cdots P_{\mu-1} x+\sum_{j=0}^{\mu-1} Q_{j} x \\
& \quad+\sum_{i=1}^{\mu-1} \sum_{j=1}^{i} P_{\mu-1} \cdots P_{j} D^{-}\left(D P_{0} \cdots P_{j} D^{-}\right)^{\prime} D P_{0} \cdots P_{i-1} Q_{i} x=G_{\mu}^{-1} q,
\end{aligned}
$$


and it shows some structure even now. By means of the decomposition

$$
I=P_{0} \cdots P_{\mu-1}+Q_{0} P_{1} \cdots P_{\mu-1}+\cdots+Q_{\mu-2} P_{\mu-1}+Q_{\mu-1}
$$

we split equation (3.1) into an equivalent system of $\mu+1$ equations corresponding to the terms involved in (3.2), all of which are projectors. Thereby we make use of the properties

$$
\begin{aligned}
& P_{0} \cdots P_{\mu-1} Q_{j}=0, j=0, \ldots, \mu-1, \\
& Q_{\mu-1} Q_{j}=0, j=0, \ldots, \mu-2, \\
& Q_{i} P_{i+1} \cdots P_{\mu-1} Q_{i}=Q_{i}, Q_{i} P_{i+1} \cdots P_{\mu-1} Q_{j}=0, j \neq i .
\end{aligned}
$$

Since the first factor on the right-hand side of

$$
Q_{i}=\left(I-\left(I-P_{0} \cdots P_{i-1}\right) Q_{i}\right) \cdot P_{0} \cdots P_{i-1} Q_{i}
$$

is nonsingular, we obtain the components $P_{0} Q_{1} x, \ldots, P_{0} \cdots P_{\mu-2} Q_{\mu-1} x$ from the components $Q_{i} x$, $i=1, \ldots, \mu-1$ and vice versa.

Let $x \in C_{D}^{1}\left(\mathcal{I}, \mathbb{R}^{m}\right)$ be a solution of (2.1) resp. (3.1). Recall the function space naturally containing the solutions to be

$$
C_{D}^{1}\left(\mathcal{I}, \mathbb{R}^{m}\right)=\left\{x \in C\left(\mathcal{I}, \mathbb{R}^{m}\right): D x \in C^{1}\left(\mathcal{I}, \mathbb{R}^{n}\right)\right\} .
$$

Define the components

$$
u=D P_{0} \cdots P_{\mu-1} x, v_{0}=Q_{0} x, v_{i}=P_{0} \cdots P_{i-1} Q_{i} x, i=1, \ldots, \mu-1,
$$

such that

$$
x=D^{-} u+v_{0}+\ldots+v_{\mu-1} .
$$

The components $u=D P_{0} \cdots P_{\mu-1} D^{-} D x, D v_{i}=D P_{0} \cdots P_{i-1} Q_{i} D^{-} D x, i=1, \ldots, \mu-1$ are continuously differentiable since $D x$ as well as the projectors in front of $D x$ are so.

Now, premultiplication of (3.1) by $P_{0} \cdots P_{\mu-1}$ (cf. (3.2)) and then by $D=D P_{0}$ leads to an explicit regular ODE for the component $u$ only (cf. Theorem 3.1 in [1]), namely

$$
u^{\prime}-\left(D P_{0} \cdots P_{\mu-1} D^{-}\right)^{\prime} u+D P_{0} \cdots P_{\mu-1} G_{\mu}^{-1} B D^{-} u=D P_{0} \cdots P_{\mu-1} G_{\mu}^{-1} q .
$$

No further components $v_{i}$ are involved in this so-called inherent regular ODE.

Further, multiplying (3.1) by $Q_{\mu-1}$ yields immediately

$$
Q_{\mu-1} x+Q_{\mu-1} G_{\mu}^{-1} B P_{0} \cdots P_{\mu-1} x=Q_{\mu-1} G_{\mu}^{-1} q .
$$

If $\mu \geq 2$, we multiply once again by $P_{0} \cdots P_{\mu-2}$, so that the resulting expressions are

$$
v_{\mu-1}=\mathcal{L}_{\mu-1} q-\mathcal{K}_{\mu-1} D^{-} u
$$

with coefficients

$$
\begin{aligned}
& \mathcal{L}_{\mu-1}=P_{0} \cdots P_{\mu-2} Q_{\mu-1} G_{\mu}^{-1} \text { for } \mu \geq 2 \\
& \mathcal{K}_{\mu-1}=P_{0} \cdots P_{\mu-2} Q_{\mu-1} G_{\mu}^{-1} B P_{0} \cdots P_{\mu-1} \text { for } \mu \geq 2 \\
& \mathcal{L}_{\mu-1}=Q_{\mu-1} G_{\mu}^{-1}, \mathcal{K}_{\mu-1}=Q_{\mu-1} G_{\mu}^{-1} B P_{0} \cdots P_{\mu-1} \text { for } \mu=1 .
\end{aligned}
$$

We avoid to write an extra subscript to indicate the coefficients to depend on $\mu$. Obviously, relation (3.6) determines the component $v_{\mu-1}$ explicitly in terms of $q$ and $u$. 
Remark 3.1 For $\mu=1$, the decomposition (3.4) and the equations (3.5), (3.6) represent the well known decoupling of an index-1 DAE into its dynamic and algebraic parts (cf. [3]):

$$
\begin{gathered}
x=D^{-} u+v_{0}, \\
u^{\prime}-R^{\prime} u+D G_{1}^{-1} B D^{-} u=D G_{1}^{-1} q, \\
v_{0}=-Q_{0} G_{1}^{-1} B P_{0} D^{-} u+Q_{0} G_{1}^{-1} q .
\end{gathered}
$$

This yields the solution representation

$$
x=\left(I-\mathcal{K}_{0}\right) D^{-} u+Q_{0} G_{1}^{-1} q, \mathcal{K}_{0}=Q_{0} G_{1}^{-1} B P_{0} .
$$

Recall $I-\mathcal{K}_{0}$ to be nonsingular, and $\left(I-\mathcal{K}_{0}\right) P_{0}$ to be the projector function along $N_{0}$ onto $S_{0}=\left\{z \in \mathbb{R}^{m}: B z \in i m G_{0}\right\}$.

For $\mu \geq 2$ we have to consider also the equations that arise by multiplying (3.1) by $Q_{0} P_{1} \cdots P_{\mu-1}$ and by $P_{0} \cdots P_{k-1} \cdot Q_{k} P_{k+1} \cdots P_{\mu-1}, k=1, \ldots, \mu-2$ (cf. (3.2)). A careful rearrangement of the involved terms (cf. Appendix B) leads to the system

$$
v_{k}=\mathcal{L}_{k} q-\mathcal{K}_{k} D^{-} u+\sum_{j=k+1}^{\mu-1} \mathcal{N}_{k j}\left(D v_{j}\right)^{\prime}+\sum_{j=k+2}^{\mu-1} \mathcal{M}_{k j} v_{j}, \quad k=0, \ldots, \mu-2
$$

with continuous coefficients $\mathcal{L}_{k}, \mathcal{K}_{k}, \mathcal{N}_{k j}$ given below, but for the $\mathcal{M}_{k j}$ we refer to Appendix B. By the system (3.6)-(3.7), for given $u$, the components $v_{\mu-1}, \ldots, v_{0}$ are successively determined in an explicit manner. While $v_{\mu-1}$ is given (cf. (3.6) by a simple assessment, the components $v_{i}, i \leq \mu-2$, depend on certain derivatives of components that are already determined. In particular, we have

$$
v_{\mu-2}=\mathcal{L}_{\mu-2} q-\mathcal{K}_{\mu-2} D^{-} u+\mathcal{N}_{\mu-2 \mu-1}\left(D v_{\mu-1}\right)^{\prime} .
$$

The coefficients in (3.7) are

$$
\begin{array}{ll}
\mathcal{L}_{0} & =Q_{0} P_{1} \cdots P_{\mu-1} G_{\mu}^{-1}, \mathcal{L}_{k}=P_{0} \cdots P_{k-1} Q_{k} P_{k+1} \cdots P_{\mu-1} G_{\mu}^{-1}, k=1, \ldots, \mu-2, \\
\mathcal{N}_{01} & =Q_{0} Q_{1} D^{-}, \mathcal{N}_{0 j}=Q_{0} P_{1} \cdots P_{j-1} Q_{j} D^{-}, j=2, \ldots, \mu-1 \\
\mathcal{N}_{k k+1} & =P_{0} \cdots P_{k-1} Q_{k} Q_{k+1} D^{-}, k=1, \ldots, \mu-2 \\
\mathcal{N}_{k j} & =P_{0} \cdots P_{k-1} Q_{k} P_{k+1} \cdots P_{j-1} Q_{j} D^{-}, j=k+2, \cdots, \mu-1, k=1, \ldots, \mu-2,
\end{array}
$$

and

$$
\begin{aligned}
\mathcal{K}_{0}= & Q_{0} P_{1} \cdots P_{\mu-1} G_{\mu}^{-1} B P_{0} \cdots P_{\mu-1}+Q_{0} P_{1} \cdots P_{\mu-1} P_{0} D^{-}\left(D P_{0} \cdots P_{\mu-1} D^{-}\right)^{\prime} D P_{0} \cdots P_{\mu-1} \\
= & Q_{0 *} P_{0} \cdots P_{\mu-1} \\
\mathcal{K}_{k}= & P_{0} \cdots P_{k-1} Q_{k} P_{k+1} \cdots P_{\mu-1} G_{\mu}^{-1} B P_{0} \cdots P_{\mu-1} \\
& \quad+P_{0} \cdots P_{k-1} Q_{k} P_{k+1} \cdots P_{\mu-1} P_{k} D^{-}\left(D P_{0} \cdots P_{\mu-1} D^{-}\right)^{\prime} D P_{0} \cdots P_{\mu-1} \\
= & P_{0} \cdots P_{k-1} Q_{k *} P_{k} \cdots P_{\mu-1}, k=1, \ldots, \mu-2
\end{aligned}
$$

with

$$
\begin{aligned}
Q_{0 *}= & Q_{0} P_{1} \cdots P_{\mu-1} G_{\mu}^{-1} B_{0}\left(I+Q_{0} P_{1} \cdots P_{\mu-1} P_{0} D^{-}\left(D P_{0} \cdots P_{\mu-1} D^{-}\right)^{\prime} D\right), \\
Q_{k *}= & Q_{k} P_{k+1} \cdots P_{\mu-1} G_{\mu}^{-1} B_{k}\left(I+Q_{k} P_{k+1} \cdots P_{\mu-1} P_{k} D^{-}\left(D P_{0} \cdots P_{\mu-1} D^{-}\right)^{\prime} D P_{0} \cdots P_{k}\right), \\
& \quad k=1, \ldots, \mu-2 .
\end{aligned}
$$


Let us mention that $Q_{k *} Q_{k}=Q_{k}, Q_{k *}^{2}=Q_{k *}, k=0, \ldots, \mu-2$. It turns out that $Q_{k *}$ is a certain extra projector function onto $N_{k}$.

Theorem 3.2 Let (2.1) be a regular DAE with tractability index $\mu$.

(i) If $x \in C_{D}^{1}\left(\mathcal{I}, \mathbb{R}^{m}\right)$ solves the $D A E(2.1)$, then

$$
\begin{aligned}
u & =D P_{0} \cdots P_{\mu-1} x \in C^{1}\left(\mathcal{I}, \mathbb{R}^{n}\right), v_{0}=Q_{0} x \in C\left(\mathcal{I}, \mathbb{R}^{m}\right), \\
v_{i} & =P_{0} \cdots P_{i-1} Q_{i} x \in C_{D}^{1}\left(\mathcal{I}, \mathbb{R}^{m}\right), i=1, \ldots, \mu-1,
\end{aligned}
$$

form a solution of the system (3.5),(3.6),(3.7), and $u=D P_{0} \cdots P_{\mu-1} D^{-} u$.

(ii) Conversely, if $u \in C^{1}\left(\mathcal{I}, \mathbb{R}^{n}\right), v_{0} \in C\left(\mathcal{I}, \mathbb{R}^{m}\right), v_{i} \in C_{D}^{1}\left(\mathcal{I}, \mathbb{R}^{m}\right), i=1, \ldots, \mu-1$, satisfy the system (3.5),(3.6),(3.7), and $u\left(t_{0}\right) \in i m\left(D P_{0} \cdots P_{\mu-1}\right)\left(t_{0}\right)$ for a $t_{0} \in \mathcal{I}$, then

$$
x=D^{-} u+v_{0}+\ldots+v_{\mu-1} \in C_{D}^{1}\left(\mathcal{I}, \mathbb{R}^{m}\right)
$$

is a solution of the DAE (2.1).

\section{Proof:}

It remains to verify the second part. Take $u, v_{0}, \ldots, v_{\mu-1}$ satisfying $(3.5),(3.6),(3.7)$. From (3.6),(3.7) we derive immediately

$$
v_{0}=Q_{0} v_{0}, v_{i}=P_{0} \cdots P_{i-1} Q_{i} v_{i}, i=1, \ldots, \mu-1 .
$$

Due to [1], Theorem 3.1 we know $u$ to satisfy

$$
u=D P_{0} \cdots P_{\mu-1} D^{-} u, u=D^{-} D u
$$

Next we put $x=D^{-} u+v_{0}+\cdots+v_{\mu-1} \in C\left(\mathcal{I}, \mathbb{R}^{m}\right)$. It follows that $D x=u+D v_{1}+\ldots+D v_{\mu-1} \in$ $C^{1}\left(\mathcal{I}, \mathbb{R}^{n}\right)$, thus $x \in C_{D}^{1}\left(\mathcal{I}, \mathbb{R}^{m}\right)$. Derive further that $D P_{0} \cdots P_{\mu-1} x=u, Q_{0} x=v_{0}, Q_{i} x=$ $Q_{i} v_{i}, P_{0} \cdots P_{i-1} Q_{i} x=v_{i}, i=1, \ldots, \mu-1$. Since the decoupling procedure for (3.1) via (3.2) leading to $(3.5),(3.6),(3.7)$ is reversible, $x$ can be checked to satisfy (3.1) in fact.

The equivalence of the DAE (2.1) to the system $(3.5),(3.6),(3.7)$ sheds some more light on the structure of the DAE.

When investigating the solvability of the DAE one can take advantage of the decoupled system. Looking at (3.6), we realize the condition

$$
D \mathcal{L}_{\mu-1} q-D \mathcal{K}_{\mu-1} D^{-} u \in C^{1}\left(\mathcal{I}, \mathbb{R}^{n}\right)
$$

as necessary for solvability. Namely, for a given solution $x \in C_{D}^{1}\left(\mathcal{I}, \mathbb{R}^{m}\right)$ the component $D P_{0} \cdots P_{\mu-2} Q_{\mu-1} x$ necessarily belongs to $C^{1}$, i.e., (3.11) is necessarily valid. On the other hand, if $q \in C\left(\mathcal{I}, \mathbb{R}^{m}\right)$ is given and we try to solve the DAE via the system $(3.5),(3.6),(3.7)$, we first obtain $u \in C^{1}\left(\mathcal{I}, \mathbb{R}^{n}\right)$ from (3.5). Assuming additionally $D \mathcal{K}_{\mu-1} D^{-}$to be continuously differentiable, we know $D v_{\mu-1}$ to belong to $C^{1}$ for $q$ with $D \mathcal{L}_{\mu-1} q \in C^{1}\left(\mathcal{I}, \mathbb{R}^{n}\right)$. Then, $v_{\mu-2}$ in (3.8) is well-defined and so on.

At this place we do not go into further technical details. We say that the right-hand side $q$ and the coefficients of the DAE (2.1) are sufficiently smooth if all terms in (3.7) are well-defined. 
Theorem 3.3 Let the DAE (2.1) be regular with tractability index $\mu$, and let the coefficients and $q$ be sufficiently smooth. Then, for each $x^{0} \in \mathbb{R}^{m}$, the IVP

$$
A(D x)^{\prime}+B x=q, x\left(t_{0}\right)-x^{0} \in N_{c a n \mu}\left(t_{0}\right)
$$

is uniquely solvable on $C_{D}^{1}\left(\mathcal{I}, \mathbb{R}^{m}\right)$.

\section{Proof:}

Take the solution $u$ of the inherent regular ODE (3.5) which satisfies the initial condition $u\left(t_{0}\right)=$ $\left(D P_{0} \cdots P_{\mu-1}\right)\left(t_{0}\right) x^{0}$. Then we determine $v_{\mu-1}, \ldots, v_{1}, v_{0}$ via $(3.6),(3.7)$. The combined function $x=D^{-} u+v_{0}+\ldots+v_{\mu-1}$ is a solution of the DAE due to Theorem 3.2. It holds that $\left(D P_{0} \cdots P_{\mu-1}\right)\left(t_{0}\right) x\left(t_{0}\right)=u\left(t_{0}\right)$, hence $\left(D P_{0} \cdots P_{\mu-1}\right)\left(t_{0}\right)\left(x\left(t_{0}\right)-x^{0}\right)=0$, i.e., (cf. Corollary 2.4) $x\left(t_{0}\right)-x^{0} \in N_{\text {canu }}\left(t_{0}\right)$.

Moreover, the homogeneous IVP $A(D x)^{\prime}+B x=0, x\left(t_{0}\right) \in N_{c a n \mu}\left(t_{0}\right)$ has the trivial solution only, and so the solution of (3.12) is unique.

Corollary 3.4 The dynamical degree of freedom of a regular DAE with tractability index $\mu$ is $d=m-\operatorname{dim}\left(N_{\text {can } \mu}\left(t_{0}\right)\right)=r_{0}+\ldots+r_{\mu-1}-(\mu-1) m$.

Remark 3.5 The initial condition in (3.12) can be rewritten as

$$
\mathfrak{C} x\left(t_{0}\right)=\mathfrak{C} x^{0}
$$

with any matrix $\mathfrak{C}$ whose nullspace coincides with $N_{\text {can } \mu}\left(t_{0}\right)$. A possible choice for that is $\mathfrak{C}=\left(D P_{0} \cdots P_{\mu-1}\right)\left(t_{0}\right)$.

\section{Refined matrix function sequences}

To the matrix function sequences given in Section 2 we introduce additional accompanying subspaces

$$
S_{i}(t)=\left\{z \in \mathbb{R}^{m}: B_{i}(t) z \in i m G_{i}(t)\right\}, t \in \mathcal{I}, i \geq 0 .
$$

Because of $i m G_{i-1} \subseteq i m G_{i}$ we can also use the descriptions

$$
\begin{aligned}
S_{i}(t) & =\left\{z \in \mathbb{R}^{m}: B_{0}(t) z \in i m G_{i}(t)\right\} \\
& =\left\{z \in \mathbb{R}^{m}: B_{0}(t) P_{0}(t) \cdots P_{i-1} z \in i m G_{i}(t)\right\}
\end{aligned}
$$

The subspaces $i m G_{i}$ are independent of the special choice of admissible projector functions (cf. Theorem 2.3), hence so are the subspaces $S_{i}$. Due to the construction it holds that $S_{0} \subseteq S_{1} \subseteq$ $\cdots \subseteq S_{i-1} \subseteq S_{i}$ and

$$
N_{0} \oplus \cdots \oplus N_{i-1} \subseteq S_{i}
$$

The first subspace $S_{0}(t)$ has a special meaning for the DAE (2.1). Namely, it is the geometric locus containing the solution values $x(t)$ of all solutions of the homogeneous equation $A(D x)^{\prime}+B x=0$. However, $S_{0}(t)$ is completely filled by those values only in the case of $\mu=1$ (cf. Remark 3.1). In the higher index cases, the subspace $S_{\text {can }}(t)$, which by definition contains all solution values and is filled by them, represents a $d$-dimensional proper subspace of $S_{0}(t)$ (cf. Corollary 3.4). However, 
at this stage we are not aware of a good description of that subspace. This would be easier if the coupling coefficients $\mathcal{K}_{1}, \ldots, \mathcal{K}_{\mu-1}$ (cf. (3.9), (3.10)) disappeared.

Applying [[7], Theorem A.13] we learn the matrix $G_{\mu}$ to be nonsingular only if the decomposition

$$
S_{\mu-1} \oplus N_{\mu-1}=\mathbb{R}^{m}
$$

is valid. Therefore, for DAEs being regular with tractability index $\mu$, we may always choose $Q_{\mu-1}$ to realize decomposition (4.3), i.e., $i m Q_{\mu-1}=N_{\mu-1}$, $\operatorname{ker} Q_{\mu-1}=S_{\mu-1}$. Because of (cf. (4.2)) $N_{0} \oplus \cdots \oplus N_{\mu-2} \subseteq S_{\mu-1}$, this choice satisfies condition (b) in Definition (2.1) at the same time. If the resulting $D P_{0} \cdots P_{\mu-1} D^{-}$is continuously differentiable, then $Q_{0}, \ldots, Q_{\mu-1}$ are admissible (supposed $Q_{0}, \ldots, Q_{\mu-2}$ are so).

Lemma 4.1 For a regular DAE (2.1) with tractability index $\mu$, let the last projector function $Q_{\mu-1}$ in an admissible sequence realize the decomposition (4.3). Then the coupling coefficient $\mathcal{K}_{\mu-1}$ in (3.7) disappears.

\section{Proof:}

Let $\tilde{Q}_{\mu-1}$ be an arbitrary projector onto $N_{\mu-1}$. Then, the expression $\tilde{Q}_{\mu-1}\left(G_{\mu-1}+\tilde{B}_{\mu-1} \tilde{Q}_{\mu-1}\right)^{-1} \tilde{B}_{\mu-1}$ is well-defined (cf. [7]), and it is a representation of the projector onto $N_{\mu-1}$ along $S_{\mu-1}$. Consequently, for $Q_{\mu-1}$ it holds that

$$
Q_{\mu-1}=Q_{\mu-1} G_{\mu}^{-1} B_{\mu-1}
$$

and further, $Q_{\mu-1} G_{\mu}^{-1} B_{\mu-1}=Q_{\mu-1} G_{\mu}^{-1} B P_{0} \cdots P_{\mu-2}$, thus $Q_{\mu-1} G_{\mu}^{-1} B P_{0} \cdots P_{\mu-1}=Q_{\mu-1} P_{\mu-1}=0$, i.e., $\mathcal{K}_{\mu-1}=0$.

\section{Example 4.2 The DAE}

$$
\left(\begin{array}{ll}
1 & 0 \\
0 & 1 \\
0 & 0
\end{array}\right)\left(\left(\begin{array}{lll}
1 & 0 & 0 \\
0 & 1 & 0
\end{array}\right) x(t)\right)^{\prime}+\left(\begin{array}{ccc}
0 & 0 & 0 \\
0 & 0 & -1 \\
-2 \alpha(t) & -1 & 0
\end{array}\right) x(t)=q(t), \quad t \in \mathcal{I}
$$

with $\alpha \in C(\mathcal{I}, \mathbb{R})$, has tractability index $\mu=2$ by Definition 2.1. Namely, a suitable matrix function sequence is given by

$$
\begin{gathered}
G_{0}=\left(\begin{array}{lll}
1 & 0 & 0 \\
0 & 1 & 0 \\
0 & 0 & 0
\end{array}\right), \quad Q_{0}=\left(\begin{array}{lll}
0 & 0 & 0 \\
0 & 0 & 0 \\
0 & 0 & 1
\end{array}\right), G_{1}=\left(\begin{array}{ccc}
1 & 0 & 0 \\
0 & 1 & -1 \\
0 & 0 & 0
\end{array}\right), \quad Q_{1}=\left(\begin{array}{lll}
0 & 0 & 0 \\
0 & 1 & 0 \\
0 & 1 & 0
\end{array}\right), \\
G_{2}=\left(\begin{array}{ccc}
1 & 0 & 0 \\
0 & 1 & -1 \\
0 & -1 & 0
\end{array}\right), \quad G_{2}^{-1}=\left(\begin{array}{ccc}
1 & 0 & 0 \\
0 & 0 & -1 \\
0 & -1 & -1
\end{array}\right), \quad D P_{1} D^{-}=\left(\begin{array}{ll}
1 & 0 \\
0 & 0
\end{array}\right) .
\end{gathered}
$$

Here we have $m=3, n=2, r_{0}=r_{1}=2, r_{2}=3$.

Since $D P_{1} G_{2}^{-1} B D^{-}=0$, the inherent regular ODE is simply $u^{\prime}=D P_{1} G_{2}^{-1} q$. Compute the coefficients

$$
\mathcal{K}_{1}=P_{0} Q_{1} G_{2}^{-1} B P_{0} P_{1}=\left(\begin{array}{ccc}
0 & 0 & 0 \\
2 \alpha & 0 & 0 \\
0 & 0 & 0
\end{array}\right), \quad \mathcal{L}_{1}=P_{0} Q_{1} G_{2}^{-1}=\left(\begin{array}{ccc}
0 & 0 & 0 \\
0 & 0 & -1 \\
0 & 0 & 0
\end{array}\right)
$$


and $D \mathcal{K}_{1} D^{-}=\left(\begin{array}{cc}0 & 0 \\ 2 \alpha & 0\end{array}\right)$, as well as the component $u=D P_{0} P_{1} x=\left(\begin{array}{c}x_{1} \\ 0\end{array}\right)$.

The necessary solvability condition (3.11) reads now in detail

$$
D \mathcal{L}_{1} q-D \mathcal{K}_{1} D^{-} u=\left(\begin{array}{c}
0 \\
-q_{3}
\end{array}\right)-\left(\begin{array}{cc}
0 & 0 \\
2 \alpha & 0
\end{array}\right) u \in C^{1}\left(\mathcal{I}, \mathbb{R}^{2}\right)
$$

It becomes clear that we cannot do with continuous $\alpha$, but we have to assume that $\alpha \in C^{1}(\mathcal{I}, \mathbb{R})$ for obtaining standard solvability assertions. Letting $q=0, \quad u$ becomes a constant function, i.e., $D \mathcal{K}_{1} D^{-} u \in C^{1}\left(\mathcal{I}, \mathbb{R}^{2}\right)$ needs necessarily $\alpha \in C^{1}(\mathcal{I}, \mathbb{R})$.

Supposing $\alpha \in C^{1}(\mathcal{I}, \mathbb{R})$ we are able to describe the set of right-hand sides that are appropriate for solvability as

$$
\left\{q \in C\left(\mathcal{I}, \mathbb{R}^{3}\right): D \mathcal{L}_{1} q \in C^{1}\left(\mathcal{I}, \mathbb{R}^{2}\right)\right\}=\left\{q \in C\left(\mathcal{I}, \mathbb{R}^{3}\right): q_{3} \in C^{1}(\mathcal{I}, \mathbb{R})\right\} .
$$

Now we refine the decoupling by choosing instead of the above $Q_{1}$ the projector $Q_{1 c}$ onto $N_{1}$ along $S_{1}=\left\{z \in \mathbb{R}^{3}: 2 \alpha z_{1}+z_{2}=0\right\}$, i.e.,

$$
Q_{1 c}=\left(\begin{array}{ccc}
0 & 0 & 0 \\
2 \alpha & 1 & 0 \\
2 \alpha & 1 & 0
\end{array}\right)
$$

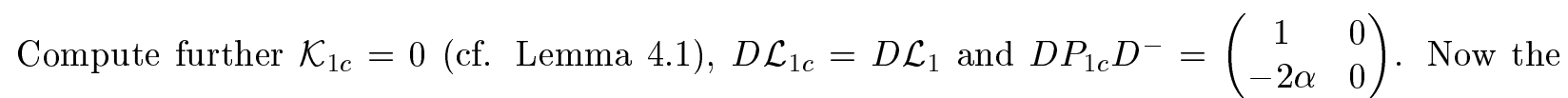
condition for $\alpha$ to belong to $C^{1}$ is put into the smoothness demands for the projector $D P_{1 c} D^{-}$or for the related subspaces $D N_{1}$ and $D S_{1}$, respectively.

Example 4.2 demonstrates the possibility of clearer solvability statements by means of a smart choice of the projectors. For general index-2 DAEs, this is confirmed in [3].

Definition 4.3 For a regular DAE (2.1) with tractability index $\mu$, the admissible projector functions $Q_{0}, \ldots, Q_{\mu-1}$ provide

(i) a fine decoupling if $\mathcal{K}_{i}=0, i=1, \ldots, \mu-1$ in (3.6), (3.7), and

(ii) a complete decoupling if $\mathcal{K}_{0}=0$ additionally.

A fine decoupling allows the precise description of the set of right-hand sides appropriate for solvability as

$$
\begin{aligned}
C^{i n d \mu}\left(\mathcal{I}, \mathbb{R}^{m}\right)= & \left\{q \in C\left(\mathcal{I}, \mathbb{R}^{m}\right): \mathcal{L}_{\mu-1} q=v_{\mu-1}, D v_{\mu-1} \in C^{1}\left(\mathcal{I}, \mathbb{R}^{n}\right)\right. \\
& \mathcal{L}_{\mu-2} q+\mathcal{N}_{\mu-2 \mu-1}\left(D v_{\mu-1}\right)^{\prime}=v_{\mu-2}, D v_{\mu-2} \in C^{1}\left(\mathcal{I}, \mathbb{R}^{n}\right), \ldots \\
& \left.\mathcal{L}_{1} q+\sum_{j=2}^{\mu-1} \mathcal{N}_{1 j}\left(D v_{j}\right)^{\prime}+\sum_{j=3}^{\mu-1} \mathcal{M}_{1 j} v_{j}=v_{1}, D v_{1} \in C^{1}\left(\mathcal{I}, \mathbb{R}^{n}\right)\right\}
\end{aligned}
$$

in particular,

$$
C^{i n d 2}\left(\mathcal{I}, \mathbb{R}^{m}\right)=\left\{q \in C\left(\mathcal{I}, \mathbb{R}^{m}\right): D P_{0} Q_{1} G_{2}^{-1} q \in C^{1}\left(\mathcal{I}, \mathbb{R}^{n}\right)\right\}
$$

For given $x \in C_{D}^{1}\left(\mathcal{I}, \mathbb{R}^{m}\right)$ the resulting $q=A(D x)^{\prime}+B x$ belongs to $C^{i n d \mu}\left(\mathcal{I}, \mathbb{R}^{m}\right)$ and, conversely, the DAE (2.1) is solvable for each $q \in C^{i n d \mu}\left(\mathcal{I}, \mathbb{R}^{m}\right)$.

As we will realize by the next theorem, a fine decoupling allows for a constructive description of the canonical subspace $S_{\text {can } \mu}(t)$, which is defined to be the geometric locus of the homogeneous DAE, i.e., $S_{\text {can } \mu}(t)=\left\{x(t): x \in C_{D}^{1}\left(\mathcal{I}, \mathbb{R}^{m}\right), A(D x)^{\prime}+B x=0\right\}$. 
Theorem 4.4 Let the $D A E$ (2.1) be regular with tractability index $\mu$, and let $Q_{0}, \ldots, Q_{\mu-1}$ provide a fine decoupling. Define $\Pi_{\text {can } \mu}=\left(I-\mathcal{K}_{0}\right) P_{0} \cdots P_{\mu-1}$.

(i) Then, for each $x^{0} \in \mathbb{R}^{m}$ and $q \in C^{\text {ind }}\left(\mathcal{I}, \mathbb{R}^{m}\right)$ the IVP (3.12) is uniquely solvable on $C_{D}^{1}\left(\mathcal{I}, \mathbb{R}^{m}\right)$

(ii) The solutions of the $D A E A(D x)^{\prime}+B x=0$ satisfy the condition $x(t) \in i m \Pi_{\text {can } \mu}(t), t \in \mathcal{I}$. For $t_{0} \in \mathcal{I}, x_{0} \in i m \Pi_{\text {can } \mu}\left(t_{0}\right)$, there is exactly one such solution that passes through $x_{0}$ at $t_{0}$. The subspaces $S_{\text {can } \mu}$ and $i m \Pi_{\text {can } \mu}$ coincide.

(iii) The decomposition

$$
S_{\text {can } \mu}(t) \oplus N_{\text {can } \mu}(t)=\mathbb{R}^{m}, \quad t \in \mathcal{I}
$$

is valid, and $\Pi_{\text {can } \mu}(t)$ is the projector that realizes (4.5).

\section{Proof:}

The first assertion is now evident as a consequence and more precise version of Theorem 3.3. We turn to the second assertion. The solutions of the homogeneous components have trivial components $v_{1}, \ldots, v_{\mu-1}$ so that $x=\left(I-\mathcal{K}_{0}\right) D^{-} u=\left(I-\mathcal{K}_{0}\right) D^{-} D P_{0} \cdots P_{\mu-1} D^{-} u=\left(I-\mathcal{K}_{0}\right) P_{0} \cdots P_{\mu-1} D^{-} u$ is a general solution representation. This implies $x(t) \in i m \Pi_{\text {can } \mu}(t), t \in \mathcal{I}$. Now write $x_{0} \in$ $i m \Pi_{\text {can } \mu}\left(t_{0}\right)$ as $x_{0}=\left(\left(I-\mathcal{K}_{0}\right) P_{0} \cdots P_{\mu-1}\right)\left(t_{0}\right) w_{0}$. Taking into account that $\mathcal{K}_{0}=Q_{0} \mathcal{K}_{0}(\mathrm{cf.}$. (3.9)), we have $\left(P_{0} \cdots P_{\mu-1}\right)\left(t_{0}\right) x_{0}=\left(P_{0} \cdots P_{\mu-1}\right)\left(t_{0}\right) w_{0}$. The IVP $A(D x)^{\prime}+B x=0, x\left(t_{0}\right)-x_{0} \in$ $N_{\text {can } \mu}\left(t_{0}\right)$ has exactly one solution, namely $x=\left(I-\mathcal{K}_{0}\right) P_{0} \cdots P_{\mu-1} D^{-} u$ with $u$ satisfying the initial condition $u\left(t_{0}\right)=\left(D P_{0} \cdots P_{\mu-1}\right)\left(t_{0}\right) x_{0}$. It follows that

$$
\begin{aligned}
x\left(t_{0}\right) & =\left(\left(I-\mathcal{K}_{0}\right) P_{0} \cdots P_{\mu-1} D^{-} D P_{0} \cdots P_{\mu-1}\right)\left(t_{0}\right) x_{0} \\
& =\left(\left(I-\mathcal{K}_{0}\right) P_{0} \cdots P_{\mu-1}\right)\left(t_{0}\right) x_{0}=\left(\left(I-\mathcal{K}_{0}\right) P_{0} \cdots P_{\mu-1}\right)\left(t_{0}\right) w_{0}=x_{0}
\end{aligned}
$$

To verify the third assertion, we observe that $\Pi_{c a n \mu}^{2}=\Pi_{c a n \mu}$ due to $P_{0} \cdots P_{\mu-1} \mathcal{K}_{0}=0$. The factor $I-\mathcal{K}_{0}$ is nonsingular, which implies

$$
k e r \Pi_{c a n \mu}=k e r P_{0} \cdots P_{\mu-1}=N_{c a n \mu}
$$

Remark 4.5 The projector function $\Pi_{\text {can } \mu}$ and subspaces $N_{\text {can } \mu}$ and $S_{\text {can } \mu}$ are characteristics of the DAE (2.1) itself and do not depend on the special choice of the fine decoupling. This is why we call them canonical. However, Theorem 4.4 describes them by means of the decoupling projectors.

There is a further benefit of fine decouplings. If there are two of them, $Q_{0}, \ldots, Q_{\mu-1}$ and $\bar{Q}_{0}, \ldots, \bar{Q}_{\mu-1}$, we derive from

$$
\left(I-\mathcal{K}_{0}\right) P_{0} \cdots P_{\mu-1}=\Pi_{c a n \mu}=\left(I-\overline{\mathcal{K}}_{0}\right) \bar{P}_{0} \cdots \bar{P}_{\mu-1}
$$

that $D P_{0} \cdots P_{\mu-1} D^{-}=D \bar{P}_{0} \cdots \bar{P}_{\mu-1} \bar{D}^{-}$must be true, i.e., the projector function $D P_{0} \cdots P_{\mu-1} D^{-}$ corresponding to the inherent regular ODE (3.5) is invariant of the special choice of the fine decoupling. In the consequence, the inherent $\mathrm{ODE}(3.5)$ and its invariant subspace $i m D P_{0} \cdots P_{\mu-1}$ are unique. 
Corollary 4.6 Let the DAE (2.1) be regular with tractability index $\mu$ and let a fine decoupling exist. Then there is a unique inherent regular ODE (3.5) being invariant of the special choice of the fine decoupling.

Finally, the question is left whether fine and complete decouplings do exist. For index 1 DAEs, each $Q_{0}$ provides a fine decoupling for trivial reasons, and $Q_{0} G_{1}^{-1} B$ provides a complete decoupling (cf. Remark 3.1). For DAEs having tractability index 2, fine as well as complete decouplings are constructed in [3]. For the case of $\mu=3$ projector functions $Q_{0}, Q_{1}, Q_{2}$ that provide a fine decoupling are presented in [8]. Unfortunately, the technical expense for the proofs in the index-3 case is rather great. Furthermore, for constant coefficient DAEs of index $\mu$, projectors $Q_{0}, \ldots, Q_{\mu-1}$ providing fine and complete decouplings are given in [6].

Recall once more the special form of the coupling coefficients

$$
\mathcal{K}_{k}=P_{0} \cdots P_{k-1} Q_{k *} P_{k} \cdots P_{\mu-1}, \quad k=1, \ldots, \mu-2,
$$

given by (3.10). This is a product of projectors and if we achieved $Q_{k *}=Q_{k}, k=1, \ldots, \mu-2$, to hold, we would have a fine decoupling. This makes us hope that the following supposition is true.

Conjecture 4.7 For a regular DAE (2.1) with tractability index $\mu$ there are projector functions $Q_{0}, \ldots, Q_{\mu-1}$ which, if the corresponding $D P_{0} \cdots P_{i} D^{-}, i=0, \ldots, \mu-1$, are continuously differentiable, provide a fine resp. a complete decoupling.

But this will probably be hard to prove, taking into account the immensely expensive proof of the $\mu=3$ case in [8]. The smoothness conditions for the $D P_{0} \cdots P_{i} D^{-}$may concern parts of the original coefficients as we have seen in Example 4.2.

In control problems (e.g. [9], [10]), a system to be controlled is said to be causal if the solutions do not depend on derivatives of the control. Consider the regular DAE with tractability index $\mu$

$$
A(D x)^{\prime}+B x=F u_{\text {control }}
$$

to be the controlled system, and $u_{\text {control }}$ to be the control. If the projector functions $Q_{0}, \ldots, Q_{\mu-1}$ provide a fine decoupling and if $F$ satisfies the condition $\left(I-P_{1} \cdots P_{\mu-1}\right) G_{\mu}^{-1} F=0$, i.e., $F=$ $G_{\mu} P_{1} \cdots P_{\mu-1} G_{\mu}^{-1} F$ (observe $G_{\mu} P_{1} \cdots P_{\mu-1} G_{\mu}^{-1}$ to be a projector function, too), then one obtains from (3.6) with $q=F u_{\text {control }}$ that $v_{\mu-1}=0$, and further, from (3.7) that $v_{\mu-2}=0, \ldots, v_{1}=$ $0, v_{0}=\mathcal{L}_{0} F u_{\text {control }}-\mathcal{K}_{0} D^{-} u=Q_{0} P_{1} \cdots P_{\mu-1} G_{\mu}^{-1} F u_{\text {control }}-\mathcal{K}_{0} D^{-} u$.

The inherent regular ODE reads now

$$
u^{\prime}-\left(D P_{0} P_{1} \cdots P_{\mu-1} D^{-}\right)^{\prime} u+D P_{0} \cdots P_{\mu-1} G_{\mu}^{-1} B D^{-} u=D P_{0} \cdots P_{\mu-1} G_{\mu}^{-1} F u_{\text {control }}
$$

Theorem 4.8 Let the DAE (4.6) be regular with tractability index $\mu$ and let $Q_{0}, \ldots, Q_{\mu-1}$ provide a fine decoupling. If

$$
F=G_{\mu} P_{1} \cdots P_{\mu-1} G_{\mu}^{-1} F
$$

is valid, then the DAE (4.6) is causal, and its solutions are given by

$$
x=\left(I-\mathcal{K}_{0}\right) D^{-} u+Q_{0} P_{1} \cdots P_{\mu-1} G_{\mu}^{-1} F u_{\text {control }},
$$

where $u$ satisfies the regular $O D E(4.7)$ and $u=D P_{0} \cdots P_{\mu-1} D^{-} u$. 
As expected, the causality condition means that the control applies to the inherent regular ODE and to the purely algebraic component.

\section{Appendix A: Proof of Theorem 2.3}

Consider two admissible sequences $G_{0}, N_{0}, Q_{0}, \ldots$, and $\bar{G}_{0}, \bar{N}_{0}, \bar{Q}_{0}, \ldots$, for the DAE (2.1). It holds that $N_{0}=\bar{N}_{0}, G_{0}=\bar{G}_{0}, B_{0}=\bar{B}_{0}, \operatorname{ker} P_{0}=\operatorname{ker} \bar{P}_{0}$. Compute $\bar{D}^{-}=\bar{P}_{0} D^{-}$

$$
\bar{G}_{1}=G_{0}+B_{0} \bar{Q}_{0}=G_{0}+B_{0} Q_{0}+B_{0} Q_{0} \bar{Q}_{0} P_{0}=G_{1} Z_{1}
$$

with the nonsingular factor

$$
Z_{1}=I+Q_{0} \bar{Q}_{0} P_{0}
$$

It results that $\bar{N}_{1}=Z_{1}^{-1} N_{1}$ and thus $\bar{N}_{0} \oplus \bar{N}_{1}=N_{0} \oplus N_{1}$ must be true. It follows that

$$
\begin{gathered}
\operatorname{ker} P_{0} P_{1}=\operatorname{ker} \bar{P}_{0} \bar{P}_{1}, \operatorname{ker} D P_{0} P_{1} D^{-}=\operatorname{ker} D \bar{P}_{0} \bar{P}_{1} \bar{D}^{-}, \\
G_{1} \bar{Q}_{1}=G_{1}\left(Z_{1}+I-Z_{1}\right) \bar{Q}_{1}=G_{1}\left(I-Z_{1}\right) \bar{Q}_{1}=-G_{1} Q_{0} \bar{Q}_{0} P_{0} \bar{Q}_{1},
\end{gathered}
$$

and further

$$
\begin{aligned}
\bar{B}_{1}= & B_{0} \bar{P}_{0}-G_{1} Z_{1} \bar{D}^{-}\left(D \bar{P}_{0} \bar{P}_{1} \bar{D}^{-}\right)^{\prime} D \\
= & B_{0} P_{0}+B_{0} Q_{0} \bar{P}_{0}-G_{1} Z_{1} \bar{D}^{-}\left(D \bar{P}_{0} \bar{P}_{1} \bar{D}^{-}\right)^{\prime} D P_{0} P_{1}-G_{1} Z_{1} \bar{D}^{-} D \bar{P}_{0} \bar{P}_{1} \bar{D}^{-}\left(D P_{0} P_{1} D^{-}\right)^{\prime} D \\
= & B_{1}+G_{1} D^{-}\left(D P_{0} P_{1} D^{-}\right)^{\prime} D+B_{0} Q_{0} \bar{P}_{0}-G_{1} Z_{1} \bar{D}^{-}\left(D \bar{P}_{0} \bar{P}_{1} \bar{D}^{-}\right)^{\prime} D P_{0} P_{1} \\
& \quad-G_{1} Z_{1} \bar{P}_{0} \bar{P}_{1} \bar{D}^{-}\left(D P_{0} P_{1} D^{-}\right)^{\prime} D
\end{aligned}
$$

Because of $G_{1}\left(I-Z_{1} \bar{P}_{0} \bar{P}_{1}\right)=G_{1}\left(I-\bar{P}_{0} \bar{P}_{1}+\left(I-Z_{1}\right) \bar{P}_{0} \bar{P}_{1}\right), G_{1}\left(I-\bar{P}_{0} \bar{P}_{1}\right)=G_{1}\left(\bar{Q}_{1}+\bar{Q}_{0} \bar{P}_{1}\right)$ and $G_{1} \bar{Q}_{1}=-G_{1} Q_{0} \bar{Q}_{0} P_{0} \bar{Q}_{1}$ we obtain the expression

$$
\bar{B}_{1}=B_{1}-G_{1} Z_{1} \bar{D}^{-}\left(D \bar{P}_{0} \bar{P}_{1} \bar{D}^{-}\right)^{\prime} D P_{0} P_{1}+G_{1} Q_{0} \mathfrak{A}_{10},
$$

where $\mathfrak{A}_{10}=\bar{P}_{0}+\left(-\bar{Q}_{0} P_{0} \bar{Q}_{1}+\bar{Q}_{0} \bar{P}_{1}-\bar{Q}_{0} P_{0} \bar{P}_{1}\right) D^{-}\left(D P_{0} P_{1} D^{-}\right)^{\prime} D$.

Next we assume the relations

$$
\begin{gathered}
\bar{G}_{i}=G_{i} Z_{i} \\
Z_{i}=\left(I+Q_{i-1} \bar{Q}_{i-1} P_{i-1}\right)\left(I+\sum_{j=0}^{i-2} Q_{j} \mathfrak{A}_{i-1 j} \bar{Q}_{i-1}\right) Z_{i-1} \\
=\left(I+Q_{i-1} \bar{Q}_{i-1} P_{i-1}+\sum_{j=0}^{i-2} Q_{j} \mathfrak{A}_{i-1 j} \bar{Q}_{i-1}\right) Z_{i-1}, \\
\bar{N}_{0} \oplus \cdots \oplus \bar{N}_{i}=N_{0} \oplus \cdots \oplus N_{i}, \\
\bar{B}_{i}=B_{i}-G_{i} Z_{i} \bar{D}^{-}\left(D \bar{P}_{0} \cdots \bar{P}_{i} \bar{D}^{-}\right)^{\prime} D P_{0} \cdots P_{i}+G_{i} \sum_{j=0}^{i-1} Q_{j} \mathfrak{A}_{i j},
\end{gathered}
$$


to be valid for $i=1, \ldots, k$. Then we know that

$$
\bar{P}_{0} \cdots \bar{P}_{i}=\bar{P}_{0} \cdots \bar{P}_{i} P_{0} \cdots P_{i}, \quad P_{0} \cdots P_{i}=P_{0} \cdots P_{i} \bar{P}_{0} \cdots \bar{P}_{i},
$$

$i m\left(Z_{i}-I\right) \subseteq N_{0} \oplus \cdots \oplus N_{i-1}, \bar{Q}_{i}=\bar{Q}_{i} \bar{P}_{0} \cdots \bar{P}_{i-1}=\bar{Q}_{i} P_{0} \cdots P_{i-1}, \quad \bar{Q}_{i} Z_{i}^{-1}=\bar{Q}_{i}$, $B_{i}\left(\bar{Q}_{i}-Q_{i}\right)=B_{i} Q_{i} \bar{Q}_{i} P_{i}$.

The special form of the coefficients $\mathfrak{A}_{i j}$ does not matter.

We show that (A.1) - (A.4) also hold true for $i=k+1$. Derive

$$
\begin{aligned}
\bar{G}_{k+1} & =G_{k} Z_{k}+\bar{B}_{k} \bar{Q}_{k}=\left(G_{k}+\bar{B}_{k} \bar{Q}_{k}\right) Z_{k} \\
& =\left(G_{k}+B_{k} \bar{Q}_{k}+G_{k} \sum_{j=0}^{k-1} Q_{j} \mathfrak{A}_{k j} \bar{Q}_{k}\right) Z_{k} \\
& =\left(G_{k}+B_{k} Q_{k}+B_{k}\left(\bar{Q}_{k}-Q_{k}\right)+G_{k} \sum_{j=0}^{k-1} Q_{j} \mathfrak{A}_{k j} \bar{Q}_{k}\right) Z_{k} \\
& =\left(G_{k+1}+B_{k} Q_{k} \bar{Q}_{k} P_{k}+G_{k} \sum_{j=0}^{k-1} Q_{j} \mathfrak{A}_{k j} \bar{Q}_{k}\right) Z_{k} \\
& =G_{k+1}\left(I+Q_{k} \bar{Q}_{k} P_{k}+\sum_{j=0}^{k-1} Q_{j} \mathfrak{A}_{k j} \bar{Q}_{k}\right) Z_{k}=G_{k+1} Z_{k+1}
\end{aligned}
$$

thus (A.1), (A.2) is given for $i=k+1$, and hence (A.3) is also true for $i=k+1$. It remains to check (A.4). Compute

$$
\begin{aligned}
& \bar{B}_{k+1}=\bar{B}_{k} \bar{P}_{k}-\bar{G}_{k+1} \bar{D}^{-}\left(D \bar{P}_{0} \cdots \bar{P}_{k+1} \bar{D}^{-}\right)^{\prime} D \bar{P}_{0} \cdots \bar{P}_{k} \\
& =\left(B_{k}-G_{k} Z_{k} \bar{D}^{-}\left(D \bar{P}_{0} \cdots \bar{P}_{k} \bar{D}^{-}\right)^{\prime} D P_{0} \cdots P_{k}+G_{k} \sum_{j=0}^{k-1} Q_{j} \mathfrak{A}_{k j}\right) \bar{P}_{k} \\
& -G_{k+1} Z_{k+1} \bar{D}^{-}\left(D \bar{P}_{0} \cdots \bar{P}_{k+1} \bar{D}^{-}\right)^{\prime} D P_{0} \cdots P_{k+1} \\
& { }_{-} G_{k+1} Z_{k+1} \bar{D}^{-} D \bar{P}_{0} \cdots \bar{P}_{k+1} \bar{D}^{-}\left(D P_{0} \cdots P_{k+1} \bar{D}^{-}\right)^{\prime} D \bar{P}_{0} \cdots \bar{P}_{k}, \\
& \bar{B}_{k+1}=B_{k} P_{k}+B_{k} Q_{k} \bar{P}_{k}-G_{k} Z_{k} \bar{D}^{-}\left(D \bar{P}_{0} \cdots \bar{P}_{k} \bar{D}^{-}\right)^{\prime} D P_{0} \cdots P_{k}+G_{k} \sum_{j=0}^{k-1} Q_{j} \mathfrak{A}_{k j} \bar{P}_{k} \\
& -G_{k+1} Z_{k+1} \bar{D}^{-}\left(D \bar{P}_{0} \cdots \bar{P}_{k+1} \bar{D}^{-}\right)^{\prime} D P_{0} \cdots P_{k+1} \\
& -G_{k+1} Z_{k+1} \bar{P}_{0} \cdots \bar{P}_{k+1} \bar{D}^{-}\left(D P_{0} \cdots P_{k+1} D^{-}\right) D \bar{P}_{0} \cdots \bar{P}_{k} \\
& \mathfrak{B}_{k+1}:=\bar{B}_{k+1}-B_{k+1}+G_{k+1} Z_{k+1} \bar{D}^{-}\left(D \bar{P}_{0} \cdots \bar{P}_{k+1} \bar{D}^{-}\right) D P_{0} \cdots P_{k+1} \\
& =G_{k+1} D^{-}\left(D P_{0} \cdots P_{k+1} D^{-}\right) D P_{0} \cdots P_{k}+B_{k} Q_{k} \bar{P}_{k} \\
& -G_{k} Z_{k} \bar{D}^{-}\left(D \bar{P}_{0} \cdots \bar{P}_{k} \bar{D}^{-}\right)^{\prime} D P_{0} \cdots P_{k}+G_{k} \sum_{j=0}^{k-1} Q_{j} \mathfrak{A}_{k j} \bar{P}_{k} \\
& -G_{k+1} Z_{k+1} \bar{P}_{0} \cdots \bar{P}_{k+1} \bar{D}^{-}\left(D P_{0} \cdots P_{k+1} D^{-}\right)^{\prime} D \bar{P}_{0} \cdots \bar{P}_{k}
\end{aligned}
$$

We have to show the representation

$$
\mathfrak{B}_{k+1}=\sum_{j=0}^{k} G_{k+1} Q_{j} \mathfrak{A}_{k+1 j}
$$


with certain coefficients $\mathfrak{A}_{k+1 j}$. The second and fourth terms on the right-hand side of (A.5) fit already into this form. Moreover, terms beginning with the expressions $G_{k}\left(Z_{k}-I\right)$ and $G_{k+1}\left(Z_{k+1}-\right.$ $I)$ are also in the right form.

Consider the remaining terms

$$
\begin{aligned}
& \tilde{\mathfrak{B}}_{k+1}:= G_{k+1} D^{-}\left(D P_{0} \cdots P_{k+1} D^{-}\right) D P_{0} \cdots P_{k}-G_{k} \bar{D}^{-}\left(D \bar{P}_{0} \cdots \bar{P}_{k} \bar{D}^{-}\right)^{\prime} D P_{0} \cdots P_{k} \\
&-G_{k+1} \bar{P}_{0} \cdots \bar{P}_{k+1} \bar{D}^{-}\left(D P_{0} \cdots P_{k+1} D^{-}\right)^{\prime} D \bar{P}_{0} \cdots \bar{P}_{k} \\
&= G_{k+1} D^{-}\left(D P_{0} \cdots P_{k+1} D^{-}\right)^{\prime} D P_{0} \cdots P_{k}-G_{k} \bar{D}^{-}\left(D \bar{P}_{0} \cdots \bar{P}_{k} \bar{D}^{-}\right)^{\prime} D P_{0} \cdots P_{k} \\
& \quad-G_{k+1} \bar{P}_{0} \cdots \bar{P}_{k+1} \bar{D}^{-}\left(D P_{0} \cdots P_{k+1} D^{-}\right)^{\prime} D P_{0} \cdots P_{k} \\
& \quad G_{k+1} \bar{P}_{0} \cdots \bar{P}_{k+1} \bar{D}^{-} D P_{0} \cdots P_{k+1} D^{-}\left(D P_{0} \cdots P_{k+1} D^{-}\right)^{\prime} D \bar{P}_{0} \cdots \bar{P}_{k}, \\
& \tilde{\mathfrak{B}}_{k+1}=G_{k+1}\left(I-\bar{P}_{0} \cdots \bar{P}_{k+1}\right) D^{-}\left(D P_{0} \cdots P_{k+1} D^{-}\right)^{\prime} D P_{0} \cdots P_{k} \\
& \quad-G_{k} \bar{D}^{-}\left(D \bar{P}_{0} \cdots \bar{P}_{k} \bar{D}^{-}\right)^{\prime} D P_{0} \cdots P_{k}-G_{k+1} \bar{P}_{0} \cdots \bar{P}_{k+1} D^{-}\left(D P_{0} \cdots P_{k} D^{-}\right)^{\prime} D \bar{P}_{0} \cdots \bar{P}_{k} .
\end{aligned}
$$

Because of $I-\bar{P}_{0} \cdots \bar{P}_{k+1}=\bar{Q}_{0} \bar{P}_{1} \ldots \bar{P}_{k+1}+\ldots+\bar{Q}_{k} P_{k+1}+\bar{Q}_{k+1}$ and $G_{k+1} \bar{Q}_{k+1}=G_{k+1}\left(Z_{k+1}+\right.$ $\left.I-Z_{k+1}\right) \bar{Q}_{k+1}=G_{k+1}\left(I-Z_{k+1}\right) \bar{Q}_{k+1}$, the first term of $\tilde{\mathfrak{B}}_{k+1}$ is already in the right form.

With

$$
\begin{aligned}
& G_{k+1} \bar{P}_{0} \cdots \bar{P}_{k+1} D^{-}\left(D P_{0} \cdots P_{k} D^{-}\right)^{\prime} D \bar{P}_{0} \cdots \bar{P}_{k} \\
& =G_{k+1} \bar{P}_{0} \cdots \bar{P}_{k+1} \bar{D}^{-} D \bar{P}_{0} \cdots \bar{P}_{k} \bar{D}^{-}\left(D P_{0} \cdots P_{k} D^{-}\right)^{\prime} D \bar{P}_{0} \cdots \bar{P}_{k} \\
& =G_{k+1} \bar{P}_{0} \cdots \bar{P}_{k+1} D^{-}\left\{\left(D \bar{P}_{0} \cdots \bar{P}_{k} \bar{D}^{-}\right)^{\prime} D \bar{P}_{0} \cdots \bar{P}_{k}-\left(D \bar{P}_{0} \cdots \bar{P}_{k} \bar{D}^{-}\right)^{\prime} D P_{0} \cdots P_{k}\right\} \\
& =-G_{k+1} \bar{P}_{0} \cdots \bar{P}_{k+1} D^{-}\left(D \bar{P}_{0} \cdots \bar{P}_{k} D^{-}\right)^{\prime} D P_{0} \cdots P_{k}
\end{aligned}
$$

we find the remaining part to be considered as

$$
\begin{aligned}
\widetilde{\tilde{B}}_{k+1} & :=-G_{k} \bar{D}^{-}\left(D \bar{P}_{0} \cdots \bar{P}_{k} \bar{D}^{-}\right)^{\prime} D P_{0} \cdots P_{k}+G_{k+1} \bar{P}_{0} \cdots \bar{P}_{k+1} \bar{D}^{-}\left(D \bar{P}_{0} \cdots \bar{P}_{k} \bar{D}^{-}\right)^{\prime} D P_{0} \cdots P_{k} \\
& \left.=\left\{G_{k+1} \bar{P}_{0} \cdots \bar{P}_{k+1}-G_{k}\right\} \bar{D}^{-} D \bar{P}_{0} \cdots \bar{P}_{k} \bar{D}^{-}\right)^{\prime} D P_{0} \cdots P_{k} .
\end{aligned}
$$

Since

$$
\begin{aligned}
G_{k+1} \bar{P}_{0} \cdots \bar{P}_{k+1}-G_{k} & =G_{k+1}-G_{k}+G_{k+1}\left(\bar{P}_{0} \ldots \bar{P}_{k+1}-I\right) \\
& =B_{k} Q_{k}+G_{k+1}\left(\bar{Q}_{0} \bar{P}_{1} \cdots \bar{P}_{k+1}+\ldots+\bar{Q}_{k} \bar{P}_{k+1}+\bar{Q}_{k+1}\right) \\
& =G_{k+1} Q_{k}+G_{k+1}\left(\bar{Q}_{0} \bar{P}_{1} \ldots \bar{P}_{k+1}+\ldots+\bar{Q}_{k} \bar{P}_{k+1}+\left(I-Z_{k+1}\right) \bar{Q}_{k+1}\right)
\end{aligned}
$$

we obtain the representation $\widetilde{\widetilde{B}}_{k+1}=\sum_{j=0}^{k} G_{k+1} Q_{j} \widetilde{\widetilde{\mathfrak{A}}}_{k+1 j}$, thus, $\tilde{\mathfrak{B}}_{k+1}=\sum_{j=0}^{k} G_{k+1} Q_{j} \tilde{\mathfrak{A}}_{k+1 j}$, and, finally (A.6). Consequently, (A.1) - (A.4) hold true for $i=k+1$ and we are done. 


\section{Appendix B: Deriving relation (3.7)}

We start with the version of the DAE (2.1) that is premultiplied by $G_{\mu}^{-1}$

$$
\begin{aligned}
P_{\mu-1} & \cdots P_{0} D^{-}(D x)^{\prime}+G_{\mu}^{-1} B P_{0} \cdots P_{\mu-1} x+\sum_{i=0}^{\mu-1} Q_{i} x \\
& +\sum_{i=0}^{\mu-1} \sum_{j=0}^{i} P_{\mu-1} \cdots P_{j} D^{-}\left(D P_{0} \cdots P_{j} D^{-}\right)^{\prime} D P_{0} \cdots P_{i-1} Q_{i} x=G_{\mu}^{-1} q
\end{aligned}
$$

and multiply by $Q_{k} P_{k+1} \cdots P_{\mu-1}$ with $0 \leq k \leq \mu-2$.

Derive

$$
\begin{aligned}
& Q_{k} P_{k+1} \cdots P_{\mu-1} P_{\mu-1} \cdots P_{0} D^{-}(D x)^{\prime}=Q_{k} P_{k+1} \cdots P_{\mu-1} P_{k} D^{-}(D x)^{\prime} \\
& =Q_{k}\left(I-Q_{k+1}-P_{k+1} Q_{k+2}-\ldots-P_{k+1} \cdots P_{\mu-2} Q_{\mu-1}\right) P_{k} D^{-}(D x)^{\prime} \\
& =-Q_{k}\left\{Q_{k+1} D^{-} D P_{0} \cdots P_{k} Q_{k+1} D^{-}(D x)^{\prime}+P_{k+1} Q_{k+2} D^{-} D P_{0} \cdots P_{k+1} Q_{k+2} D^{-}(D x)^{\prime}\right. \\
& \left.\quad \quad+\cdots+P_{k+1} \cdots P_{\mu-2} Q_{\mu-1} D^{-} D P_{0} \cdots P_{\mu-2} Q_{\mu-1} D^{-}(D x)^{\prime}\right\} \\
& \quad \quad+Q_{k} Q_{k+1} D^{-}\left(D P_{0} \cdots P_{k} Q_{k+1} x\right)^{\prime}+Q_{k} Q_{k+1} D^{-}\left(D P_{0} \cdots P_{k} Q_{k+1} D^{-}\right)^{\prime} D x \\
& \quad-Q_{k} P_{k+1} Q_{k+2} D^{-}\left(D P_{0} \cdots P_{k+1} Q_{k+2} x\right)^{\prime}+Q_{k} P_{k+1} Q_{k+2} D^{-}\left(D P_{0} \cdots P_{k+1} Q_{k+2} D^{-}\right)^{\prime} D x \\
& \quad-\ldots \\
& \quad-Q_{k} P_{k+1} \cdots P_{\mu-2} Q_{\mu-1} D^{-}\left(D P_{0} \cdots P_{\mu-2} Q_{\mu-1} x\right)^{\prime} \\
& \quad \quad+Q_{k} P_{k+1} \cdots P_{\mu-2} Q_{\mu-1} D^{-}\left(D P_{0} \cdots P_{\mu-2} Q_{\mu-1} D^{-}\right)^{\prime} D x \\
& =-Q_{k} Q_{k+1} D^{-}\left(D P_{0} \cdots P_{k} Q_{k+1} x\right)^{\prime}-Q_{k} P_{k+1} Q_{k+2} D^{-}\left(D P_{0} \cdots P_{k+1} Q_{k+2} x\right)^{\prime} \\
& \quad-\ldots-Q_{k} P_{k+1} \cdots P_{\mu-2} Q_{\mu-1} D^{-}\left(D P_{0} \cdots P_{\mu-2} Q_{\mu-1} x\right)^{\prime} \\
& \quad+Q_{k}\left\{Q_{k+1} D^{-}\left(D P_{0} \cdots P_{k} Q_{k+1} D^{-}\right)+P_{k+1} Q_{k+2} D^{-}\left(D P_{0} \cdots P_{k+1} Q_{k+2} D^{-}\right)^{\prime}\right. \\
& \left.\quad+\ldots+P_{k+1} \cdots P_{\mu-2} Q_{\mu-1} D^{-}\left(D P_{0} \cdots P_{\mu-2} Q_{\mu-1} D^{-}\right)^{\prime}\right\} D x
\end{aligned}
$$

and decompose $D x=D\left(P_{0} \cdots P_{\mu-1}+P_{0} \cdots P_{\mu-1} Q_{\mu-1}+\ldots+P_{0} Q_{1}\right) x$. Recall once more that $Q_{k} P_{k+1} \cdots P_{\mu-1} Q_{k}=Q_{k}, Q_{k} P_{k+1} \cdots P_{\mu-1} Q_{j}=0$ for $j \neq k$.

The equation resulting from (B.1) is

$$
\begin{aligned}
& -Q_{k} Q_{k+1} D^{-}\left(D P_{0} \cdots P_{k} Q_{k+1} x\right)^{\prime}-\ldots-Q_{k} P_{k+1} \cdots P_{\mu-2} Q_{\mu-1} D^{-}\left(D P_{0} \cdots P_{\mu-2} Q_{\mu-1} x\right)^{\prime} \\
& +Q_{k}\left\{Q_{k+1} D^{-}\left(D P_{0} \cdots P_{k} Q_{k+1} D^{-}\right)^{\prime}+\ldots+P_{k+1} \cdots P_{\mu-2} Q_{\mu-1} D^{-}\left(D P_{0} \cdots P_{\mu-2} Q_{\mu-1} D^{-}\right)^{\prime}\right\} \times \\
& \quad \times\left\{D P_{0} \cdots P_{\mu-1} x+D P_{0} \cdots P_{\mu-2} Q_{\mu-1} x+\ldots+D P_{0} Q_{1} x\right\}+Q_{k} P_{k+1} \cdots P_{\mu-1} G_{\mu}^{-1} B P_{0} \cdots P_{\mu-1} x+Q_{k} x \\
& +\sum_{i=1}^{\mu-1} \sum_{j=1}^{i} Q_{k} P_{k+1} \cdots P_{\mu-1} P_{\mu-1} \cdots P_{j} D^{-}\left(D P_{0} \cdots P_{j} D^{-}\right)^{\prime} D P_{0} \cdots P_{i-1} Q_{i} x=Q_{k} P_{k+1} \cdots P_{\mu-1} G_{\mu}^{-1} q .
\end{aligned}
$$

Next, if $k>0$, we multiply once more by $P_{0} \cdots P_{k-1}$.

Using the denotation $v_{j}=P_{0} \cdots P_{j-1} Q_{j} x, j=1, \ldots, \mu-1, v_{0}=Q_{0} x, u=D P_{0} \cdots P_{\mu-1} x$, we rearrange things to

$$
v_{k}=\mathcal{L}_{k} q+\mathcal{K}_{k} D^{-} u+\sum_{j=k+1}^{\mu-1} \mathcal{N}_{k j}\left(D v_{j}\right)^{\prime}+\sum_{j=1}^{\mu-1} \mathcal{M}_{k j} v_{j}
$$


with coefficients (cf. Section 3)

$$
\begin{aligned}
\mathcal{L}_{k}= & P_{0} \cdots P_{k-1} Q_{k} P_{k+1} \cdots P_{\mu-1} G_{\mu}^{-1}, \\
\mathcal{N}_{k k+1}= & P_{0} \cdots P_{k-1} Q_{k} Q_{k+1} D^{-}, N_{k j}=P_{0} \cdots P_{k-1} Q_{k} P_{k+1} \cdots P_{j-1} Q_{j} D^{-}, j=k+2, \ldots, \mu-1, \\
\mathcal{K}_{k}= & -P_{0} \cdots P_{k-1} Q_{k} P_{k+1} \cdots P_{\mu-1} G_{\mu}^{-1} B P_{0} \cdots P_{\mu-1} \\
& -P_{0} \cdots P_{k-1} Q_{k}\left\{Q_{k+1} D^{-}\left(D P_{0} \cdots P_{k} Q_{k+1} D^{-}\right)^{\prime}+P_{k+1} Q_{k+2} D^{-}\left(D P_{0} \cdots P_{k+1} Q_{k+2} D^{-}\right)^{\prime}\right. \\
& \left.+\ldots+P_{k+1} \cdots P_{\mu-2} Q_{\mu-1} D^{-}\left(D P_{0} \cdots P_{\mu-2} Q_{\mu-1} D^{-}\right)^{\prime}\right\} D P_{0} \cdots P_{\mu-1} \\
= & -P_{0} \cdots P_{k-1} Q_{k} P_{k+1} \cdots P_{\mu-1} G_{\mu}^{-1} B P_{0} \cdots P_{\mu-1} \\
& +P_{0} \cdots P_{k-1} Q_{k}\left\{Q_{k+1}+P_{k+1} Q_{k+2}+\ldots\right. \\
& \left.+P_{k+1} \cdots P_{\mu-2} Q_{\mu-1}\right\} D^{-}\left(D P_{0} \cdots P_{\mu-1} D^{-}\right)^{\prime} D P_{0} \cdots P_{\mu-1} \\
= & -P_{0} \cdots P_{k-1} Q_{k} P_{k+1} \cdots P_{\mu-1} G_{\mu}^{-1} B P_{0} \cdots P_{\mu-1} \\
& -P_{0} \cdots P_{k-1} Q_{k} P_{k+1} \cdots P_{\mu-1} P_{k} D^{-}\left(D P_{0} \cdots P_{\mu-1} D^{-}\right)^{\prime} D P_{0} \cdots P_{\mu-1}, \\
= & -P_{0} \cdots P_{k-1} Q_{k}\left\{Q_{k+1} D^{-}\left(D P_{0} \cdots P_{k} Q_{k+1} D^{-}\right)^{\prime}+P_{k+1} Q_{k+2} D^{-}\left(D P_{0} \cdots P_{k+1} Q_{k+2} D^{-}\right)^{\prime}\right. \\
& \left.+\ldots+P_{k+1} \cdots P_{\mu-2} Q_{\mu-1} D^{-}\left(D P_{0} \cdots P_{\mu-2} Q_{\mu-1} D^{-}\right)^{\prime}\right\} D P_{0} \cdots P_{j-1} Q_{j} \\
& -\sum_{l=1}^{i} P_{0} \cdots P_{k-1} Q_{k} P_{k+1} \cdots P_{\mu-1} P_{\mu-1} \cdots P_{l} D^{-}\left(D P_{0} \cdots P_{l} D^{-}\right)^{\prime} D P_{0} \cdots P_{j-1} Q_{j}, \\
\mathcal{M}_{k j}, &
\end{aligned}
$$

if $k>0$, and corresponding expressions (starting with $Q_{0} P_{1} \cdots P_{\mu-1}$ ) for $k=0$.

Taking a closer look at the coefficient $\mathcal{M}_{k j}$ we show that $\mathcal{M}_{k j}=0$ for $j=1, \ldots, k+1$. Namely, for $1 \leq j \leq k$ it holds that

$$
\begin{aligned}
\mathcal{M}_{k j}= & -P_{0} \cdots P_{k-1} Q_{k} P_{k+1} \cdots P_{\mu-1} P_{k} D^{-}\left(D P_{0} \cdots P_{j-1} Q_{j} D^{-}\right)^{\prime} D P_{0} \cdots P_{j-1} Q_{j} \\
& -\sum_{l=1}^{i} P_{0} \cdots P_{k-1} Q_{k} P_{k+1} \cdots P_{\mu-1} P_{k} D^{-}\left(D P_{0} \cdots P_{l} D^{-}\right) D P_{0} \cdots P_{j-1} Q_{j} \\
= & -P_{0} \cdots P_{k-1} Q_{k} P_{k+1} \cdots P_{\mu-1} P_{k} D^{-}\left(D P_{0} \cdots P_{j-1} Q_{j} D^{-}\right) D P_{0} \cdots P_{j-1} Q_{j} \\
& -P_{0} \cdots P_{k-1} Q_{k} P_{k+1} \cdots P_{\mu-1} P_{k} D^{-}\left(D P_{0} \cdots P_{j} D^{-}\right)^{\prime} D P_{0} \cdots P_{j-1} Q_{j} \\
= & -P_{0} \cdots P_{k-1} Q_{k} P_{k+1} \cdots P_{\mu-1} P_{k} D^{-}\left(D P_{0} \cdots P_{j} D^{-}\right)^{\prime} D P_{0} \cdots P_{j-1} Q_{j}=0 .
\end{aligned}
$$

Furthermore, compute

$$
\begin{aligned}
\mathcal{M}_{k k+1}= & -P_{0} \cdots P_{k-1} Q_{k}\left\{Q_{k+1} D^{-}\left(D P_{0} \cdots P_{k} Q_{k+1} D^{-}\right)^{\prime}+P_{k+1} Q_{k+2} D^{-}\left(D P_{0} \cdots P_{k+1} Q_{k+2} D^{-}\right)^{\prime}\right. \\
& \left.+\ldots+P_{k+1} \cdots P_{\mu-2} Q_{\mu-1} D^{-}\left(D P_{0} \cdots P_{\mu-2} Q_{\mu-1} D^{-}\right)^{\prime}\right\} D P_{0} \cdots P_{k} Q_{k+1} \\
& -\sum_{l=1}^{k+1} P_{0} P_{k-1} Q_{k} P_{k+1} \cdots P_{\mu-1} P_{\mu-1} \cdots P_{l} D^{-}\left(D P_{0} \cdots P_{l} D^{-}\right)^{\prime} D P_{0} \cdots P_{k} Q_{k+1} \\
= & -P_{0} \cdots P_{k-1} Q_{k} Q_{k+1} D^{-}\left(D P_{0} \cdots P_{k+1} Q_{k+1} D^{-}\right)^{\prime} D P_{0} \cdots P_{k} Q_{k+1} \\
& +P_{0} \cdots P_{k-1} Q_{k}\left\{Q_{k+1} D^{-}+\cdots+P_{k+1} \cdots P_{\mu-2} Q_{\mu-1} D^{-}\right\}\left(D P_{0} \cdots P_{k} Q_{k+1} D^{-}\right)^{\prime} D P_{0} \cdots P_{k} Q_{k+1} \\
& -\sum_{l=1}^{k} P_{0} \cdots P_{k-1} Q_{k} P_{k+1} \cdots P_{\mu-1} P_{k} D^{-}\left(D P_{0} \cdots P_{l} D^{-}\right) D P_{0} \cdots P_{k} Q_{k+1} \\
& -P_{0} \cdots P_{k-1} Q_{k} P_{k+1} \cdots P_{\mu-1} D^{-}\left(D P_{0} \cdots P_{k+1} D^{-}\right) D P_{0} \cdots P_{k} Q_{k+1},
\end{aligned}
$$


that is,

$$
\begin{aligned}
\mathcal{M}_{k k+1}= & -P_{0} \cdots P_{k-1} Q_{k} P_{k+1} \cdots P_{\mu-1} P_{k} D^{-}\left(D P_{0} \cdots P_{k} Q_{k+1} D^{-}\right)^{\prime} D P_{0} \cdots P_{k} Q_{k+1} \\
& -P_{0} \cdots P_{k-1} Q_{k} P_{k+1} \cdots P_{\mu-1} P_{k} D^{-}\left(D P_{0} \cdots P_{k} P_{k+1} D^{-}\right)^{\prime} D P_{0} \cdots P_{k} Q_{k+1} \\
= & -P_{0} \cdots P_{k-1} Q_{k} P_{k+1} \cdots P_{\mu-1} D^{-}\left(D P_{0} \cdots P_{k} D^{-}\right)^{\prime} D P_{0} \cdots P_{k} Q_{k+1} \\
& +P_{0} \cdots P_{k-1} Q_{k} D^{-}\left(D P_{0} \cdots P_{k} Q_{k+1} D^{-}\right)^{\prime} D P_{0} \cdots P_{k} Q_{k+1} \\
= & -P_{0} \cdots P_{k-1} Q_{k}\left\{P_{k+1} \cdots P_{\mu-1}-I\right) D^{-}\left(D P_{0} \cdots P_{k} D^{-}\right)^{\prime} D P_{0} \cdots P_{k} Q_{k+1} \\
& -P_{0} \cdots P_{k-1} Q_{k} D^{-}\left(D P_{0} \cdots P_{k} P_{k+1} D^{-}\right)^{\prime} D P_{0} \cdots P_{k} Q_{k+1}, \\
\mathcal{M}_{k k+1}= & -P_{0} \cdots P_{k-1} Q_{k} P_{k+1} \cdots P_{\mu-1} P_{k} D^{-}\left(D P_{0} \cdots P_{k} D^{-}\right)^{\prime} D P_{0} \cdots P_{k} Q_{k+1} \\
& +P_{0} \cdots P_{k-1} Q_{k} P_{0} \cdots P_{k} P_{k+1} D^{-}\left(D P_{0} \cdots P_{k} Q_{k+1} D^{-}\right)^{\prime} D .
\end{aligned}
$$

In the last formula, both expressions on the right-hand side vanish, i.e., $\mathcal{M}_{k k+1}=0$. Hence, formula (B.2) simplifies to formula (3.7), since $\mathcal{M}_{k 1}, \ldots \mathcal{M}_{k k+1}$ disappear.

\section{References}

[1] R. März, The index of linear differential algebraic equations with properly stated leading terms. Results in Mathematics 42(2002), 308-338.

[2] P.J. Rabier, W.C. Rheinboldt, Theoretical and numerical analysis of differential algebraic equations. Handbook of Numerical Analysis, Vol VIII, Elsevier Publ. Amsterdam, 2002.

[3] K. Balla, R. März, A unified approach to linear differential algebraic equations and their adjoints. Zeitschrift f. Analysis und ihre Anwendungen 21(2002)3, 783-802.

[4] R. März, Differential algebraic systems anew. Applied Numerical Mathematics 42(2002), 315-335.

[5] G. Zielke, Motivation und Darstelllung von verallgemeinerten Matrixinversen. Beiträge zur Numerischen Mathematik 7(1979), 177-218.

[6] R. März, Canonical projectors for linear differential algebraic equations. Computers Math. Applic. 31(1996) 4/5, 121-135.

[7] E. Griepentrog, R. März, Differential-algebraic equations and their numerical treatment. Teubner, Leipzig, 1986.

[8] I. Schumilina, Index-3 DAEs with properly stated leading term. Humboldt-Universität zu Berlin Institut für Mathematik, Preprint 2001-20, 2001.

[9] L. Dai, Singular control systems. Lect. Notes Control Inf. Sci. 118, Springer, Berlin 1989.

[10] P.C. Müller, Kausale und nichtkausale Descriptorsysteme. Z. Angew. Math. Mech. 77(1997), Suppl. 1, 231-232

Roswitha März

Humboldt-Universität zu Berlin

Institut für Mathematik

Unter den Linden 6

D-10099 Berlin

Eingegangen am 6. Dezember 2002 